\title{
Gly369Cys mutation in mouse FGFR3 causes achondroplasia by affecting both chondrogenesis and osteogenesis
}

\author{
Lin Chen, ${ }^{1}$ Rivka Adar, ${ }^{2}$ Xiao Yang, ${ }^{1}$ Efrat O. Monsonego, ${ }^{2}$ Cuiling Li, ${ }^{1}$ \\ Peter V. Hauschka, ${ }^{3}$ Avner Yayon, ${ }^{2}$ and Chu-Xia Deng ${ }^{1}$ \\ ${ }^{1}$ Genetics of Development and Disease Branch, National Institute of Diabetes, Digestive and Kidney Diseases, \\ National Institutes of Health, Bethesda, Maryland 20892, USA \\ ${ }^{2}$ Department of Molecular Cell Biology, Weizmann Institute of Science, Rehovot 76100, Israel \\ ${ }^{3}$ Departments of Orthopaedic Surgery and Oral Biology, Harvard Schools of Medicine and Dental Medicine, \\ Children's Hospital, Boston, Massachusetts 02115, USA
}

Address correspondence to: Chu-Xia Deng, Genetics of Development and Disease Branch, National Institute of Diabetes, Digestive and Kidney Diseases, National Institutes of Health, 10/9N105, 10 Center Drive, Bethesda, Maryland 20892, USA. Phone: (301) 402-7225; Fax: (301) 480-1135; Email: ChuxiaD@BDG10.NIDDK.NIH.Gov.

Received for publication March 2, 1999, and accepted in revised form October 19, 1999.

\begin{abstract}
Missense mutations in fibroblast growth factor receptor 3 (FGFR3) result in several human skeletal dysplasias, including the most common form of dwarfism, achondroplasia. Here we show that a glycine-to-cysteine substitution at position 375 (Gly375Cys) in human FGFR3 causes ligand-independent dimerization and phosphorylation of FGFR3 and that the equivalent substitution at position 369 (Gly369Cys) in mouse FGFR3 causes dwarfism with features mimicking human achondroplasia. Accordingly, homozygous mice were more severely affected than heterozygotes. The resulting mutant mice exhibited macrocephaly and shortened limbs due to retarded endochondral bone growth and premature closure of cranial base synchondroses. Compared with their wild-type littermates, mutant mice growth plates shared an expanded resting zone and narrowed proliferating and hypertrophic zones, which is correlated with the activation of Stat proteins and upregulation of cell-cycle inhibitors. Reduced bone density is accompanied by increased activity of osteoclasts and upregulation of genes that are related to osteoblast differentiation, including osteopontin, osteonectin, and osteocalcin. These data reveal an essential role for FGF/FGFR3 signals in both chondrogenesis and osteogenesis during endochondral ossification.
\end{abstract}

J. Clin. Invest. 104:1517-1525 (1999).

\section{Introduction}

There are 2 major modes of bone development, intramembranous ossification and endochondral ossification. The former occurs when mesenchymal precursor cells directly differentiate into bone-forming osteoblasts, a process by which all flat bones are formed. The latter entails the conversion of an initial cartilage template into bone and is responsible for generating the long bones of the skeleton (1). Fibroblast growth factor receptors (FGFRs) have been implicated in both processes of bone formation. Mutations in 3 FGF receptors, FGFR1-3, result in craniosynostoses, including Pfeiffer, Crouzon, Apert, JacksonWeiss, and Beare-Stevenson cutis gyrata syndromes (2-4). Mutations in FGFR3 were also found to cause several skeletal dysplasias that involve primarily long bone growth, including achondroplasia (ACH), hypochondroplasia $(\mathrm{HCH})$, and thanatophoric dysplasia (TD) (5-9). ACH is the most common form of dwarfism, with a frequency of approximately 1 in 20,000 live births. Patients with ACH exhibit a characteristic phenotype of rhizomelic dwarfism, relative macrocephaly, exaggerated lumbar lordosis, and min- imal chondrocyte proliferation in the growth plate cartilage of long bones. ACH is most frequently caused by a glycine-to-arginine substitution at position 380 (Gly380Arg), and can also be caused by a change from glycine to cysteine at position 375 (Gly375Cys) $(5,6,10)$.

The effect of the Gly380Arg mutation on functions of FGFR3 has been investigated extensively. The Gly380Arg mutation causes ligand-independent activation of FGFR3 in vitro and results in dwarfism in vivo (11-14). Little is known, however, about the possible effect of the Gly375Cys mutation on the functions of FGFR3. In this study, we examined the effect of the Gly375Cys mutation using both in vitro and in vivo approaches. Our data indicated that the Gly375Cys mutation caused activation of FGFR3 by inducing ligand-independent dimerization of the receptor in cultured cells. To study further the function of FGFR3 in bone growth, and to create a mouse model for the FGFR3-related inherited skeletal disorders, we introduced a Gly369Cys mutation, which corresponds to the Gly375Cys mutation in human, into the mouse genome using gene targeting. Mice carrying 
a



b
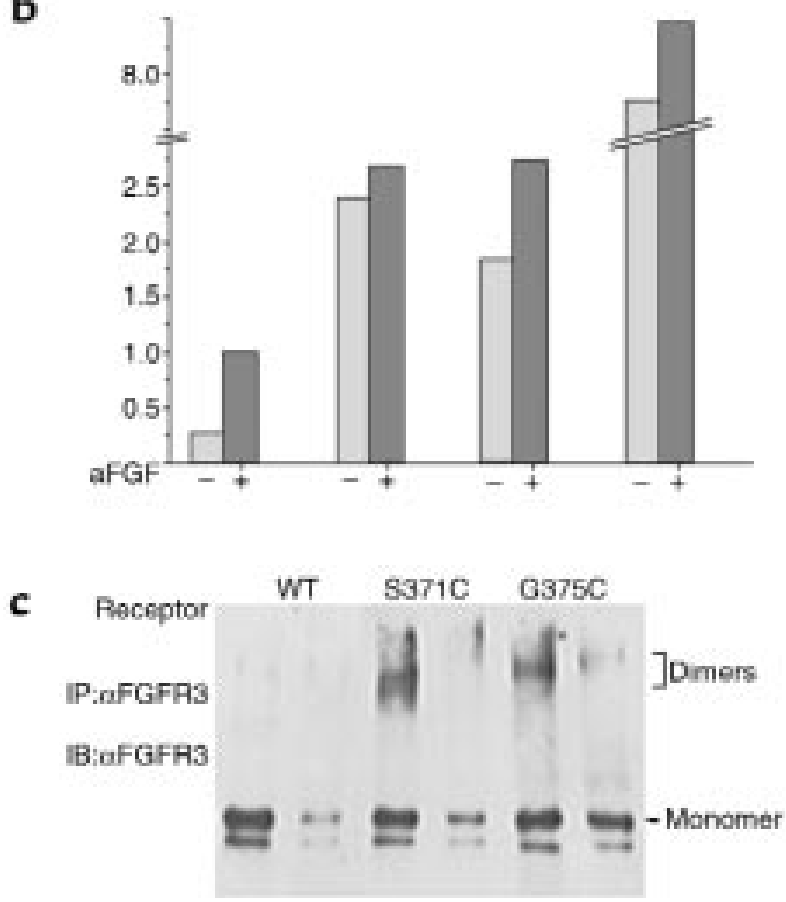

Figure 1

Introduction of Gly369Cys mutation into the mouse Fofr3 locus. (a) Targeting construct, pFgfr3-Gly369Cys, contained the Gly369Cys mutation in exon 10 and a pLoxpneo gene in intron 10 of the Fgfr3 gene. Of $120 \mathrm{G} 418^{r} / \mathrm{FIAU}$ clones examined by Southern blot analysis using a $5^{\prime}$ flanking probe (probe 1 ), 5 clones showed an extra fragment of approximately $11 \mathrm{~kb}$ upon Notl + EcoRV digestion (c). The targeting events were confirmed by Xbal +EcoRV digestion using a $3^{\prime}$ internal probe (probe 2). Three of them had the point mutation cotransferred as indicated by sequencing (unpublished observations). (b) Removal of the pLoxpneo gene by breeding with Ella-cre transgenic mice. The Cre-mediated deletion was genotyped by PCR as described in the Methods section.

the Gly369Cys mutation exhibited skeletal dysplasia similar to human ACH. Phenotypic analysis of mutant mice revealed that FGF/FGFR3 signals affect both chondrogenesis and osteogenesis by regulating Stat proteins and cell-cycle inhibitors, and the activities of chondrocytes, osteoclasts, and osteoblasts during endochondral ossification.

\section{Methods}

In vitro dimerization and activation of FGFR3.293T cells were transfected with $5 \mu \mathrm{g}$ of the different constructs using the calcium-phosphate method. Two days after transfection, cells were incubated for 2 hours at $4^{\circ} \mathrm{C}$ in the absence or presence of $50 \mathrm{ng} / \mathrm{mL}$ of acidic FGF
(aFGF). After chemical crosslinking, cells were lysed, immunoprecipitated with anti-FGFR3 $\mathrm{COOH}$-terminus antibodies (Santa Cruz Biotechnology, Santa Cruz, California, USA) and probed on an immunoblot with a polyclonal antibody against the kinase domain of FGFR3 (Santa Cruz Biotechnology). To check for receptor phosphorylation, cells were activated for 9 minutes with $50 \mathrm{ng} / \mathrm{mL}$ aFGF, lysed, immunoprecipitated with antiphosphotyrosine antibodies (mAb 4G10), and blotted with the antibody against the kinase domain of FGFR3.

Site-directed mutagenesis and targeting vector construction. The Gly369Cys mutation was introduced into exon 10 of the mouse Fgfr3 gene using an oligonucleotide, $5^{\prime}$-gcagcgtgtacgcaTgcgtcctcagctacgg- $3^{\prime}$, and a standard site-directed mutagenesis protocol ( $\mathrm{T}$ indicates the point mutation introduced). The targeting vector was constructed using Fgfr3 genomic DNA isolated previously (15). A 2.8-kb EcoRI-SmaI fragment containing the Gly369Cys mutation was inserted into pLoxpneo vector (16) through its EcoRI and Bam HI sites. The resulting plasmid was digested with $\mathrm{HpaI}$ and NotI followed by insertion of a 3.9-kb SmaI-XbaI fragment. The finished targeting construct, $p F g f r 3-$ G369C, is shown in Figure 1a.

Homologous recombination in embyronic stem cells and generation of germline chimeras. TC1 embryonic stem (ES) cells (15) were transfected with NotI-digested pFgfr3G369C. Genomic DNAs from G418- and FIAU-resistant ES clones were digested with $N o t \mathrm{I}+E c o \mathrm{RV}$ or $\mathrm{Xba \textrm {I }}$ and then probed with the $5^{\prime}$ flanking (Figure 1c), or $3^{\prime}$ internal fragments (unpublished observations) specific to the Fgfr3 sequence. Blastocyst injection and screening for germline transmission were carried out following standard procedures.

Genotype analysis. Mice with germline transmission of Gly369Cys were crossed with EIIa-Cre mice (17) to delete the neo gene. After removal of the neo gene, genotypes were determined by PCR using primers 1 : (5'-CCGGGGGAAAGCTTGAAAA- $3^{\prime}$ ), and 2 (5'-TGTAAAAGGGGTGGGGTGGTAG-3'). This pair of primers flanks the SmaI site and amplifies 534 bp in wildtype and $594 \mathrm{bp}$ in Fgfr $3^{369 /+}$ mice due to the presence of a loxP site.

Skeleton staining. Animals were euthanized by asphyxiation in $\mathrm{CO}_{2}$. After removing their skins, the carcasses were eviscerated, fixed in 95\% ethanol, stained with Alizarin Red S and Alcian Blue, cleared by $\mathrm{KOH}$ treatment, and stored in glycerol as described elsewhere (15).

Histology and antibody staining. Histological sections were prepared from selected tissues fixed in $10 \%$ formalin, decalcified with $15 \%$ EDTA at $4{ }^{\circ} \mathrm{C}$ for 1 week, and embedded in paraffin. Five-micron sections were either stained with hematoxylin and eosin (H\&E) or were subjected to immunohistochemical staining using antibodies specific to FGFR3 (D. Ornitz, Washington University), P16 and P19 (Y. Xiong, University of North Carolina), Stat 1 (Santa Cruz Biotechnology, Santa Cruz, California, USA), Stat5b, Stat5a (L. Hen- 
nighausen, National Institutes of Health) and osteocalcin. Detection of primary antibodies was performed with the Histomouse SP Kit (Zymed Laboratories Inc., South San Francisco, California, USA).

In situ bybridization and thymidine labeling. In situ hybridization was performed using standard procedures (18). The Fgfr3 probe is a 562-bp NcoI-EcoRI fragment from nucleotides 226-788 of the FGFR-3 cDNA (19). Other probes are colX (B. Olsen, Harvard Medical School), osteocalcin (G. Karsenty, Baylor College of Medicine), osteonectin, and osteopontin (L. Fisher, National Institutes of Health). $\left[{ }^{3} \mathrm{H}\right]$ Thymidine (Amersham Pharmacia Biotech, Arlington Heights, Illinois, USA) was intraperitoneally injected at a dosage of $20 \mu \mathrm{Ci} / \mathrm{g}$. Animals were sacrificed 2 hours after injection. Slides were dipped in emulsion (Kodak NTB-2; Eastman Kodak Company, Rochester, New York, USA) and exposed for 4-15 days before developing.

\section{Results}

Gly375Cys mutation in buman FGFR3 causes ligand-independent dimerization and activation. To gain insight into the mechanism underlying the Gly375Cys mutation in human achondroplasia, we analyzed the capacity of this mutant receptor to undergo ligand-dependent and independent dimerization and activation (Figure 2). While wild-type receptor required ligand for autophosphorylation, Gly375Cys FGFR3, like Ser371Cys and Lys650Glu FGFR3 (which result in TDI and TDII, respectively), was constitutively phosphorylated (Figure 2 , a and b). Quantitative analysis by densitometry of a typical experiment (Figure $2 \mathrm{~b}$ ), normalized for receptor levels, indicated that the Gly375Cys mutant, like the wild-type receptor, shared a further, ligand-dependent activation as well. The substitution of Gly by Cys has created, as expected, ligand-independent receptor dimers, most likely due to the unpaired Cys that now pairs with Cys in another mutant receptor (Figure 2c). A Gly369Cys mutation in murine FGFR3 results in dwarf mice. Gly 375 in human FGFR3 corresponds to Gly 369 in the mouse protein. To study the effect of that mutation in vivo, we introduced the Gly369Cys mutation into the mouse genome using a cotransfer-type targeting construct (20), pFgfr3-G369C (Figure 1a). Germline transmission was obtained from targeted ES cells, which were identified by Southern blot (Figure 1c). Because the presence of the pLoxpneo gene in intron 10 was found to block normal splicing of the Fgfr3 gene (data not shown), leading to phenotypes resembling those of FGFR3-null mice reported previously $(15,21)$, we crossed heterozygous mice (Fgfr3neo369/+) with EIIa-cre mice (17) to excise the pLoxpneo gene from the germline (Figure 1b). After removal of the neo gene, the mutant allele was found to be expressed at the same level as the wild-type allele by Northern blot analysis (data not shown). All data presented below are from mice without the neo gene.

Mice heterozygous for the Gly369Cys mutation $\left(\right.$ Fgfr $\left.3^{369 /+}\right)$ were smaller and displayed a significantly shorter tail length than their wild-type littermates (Fig-
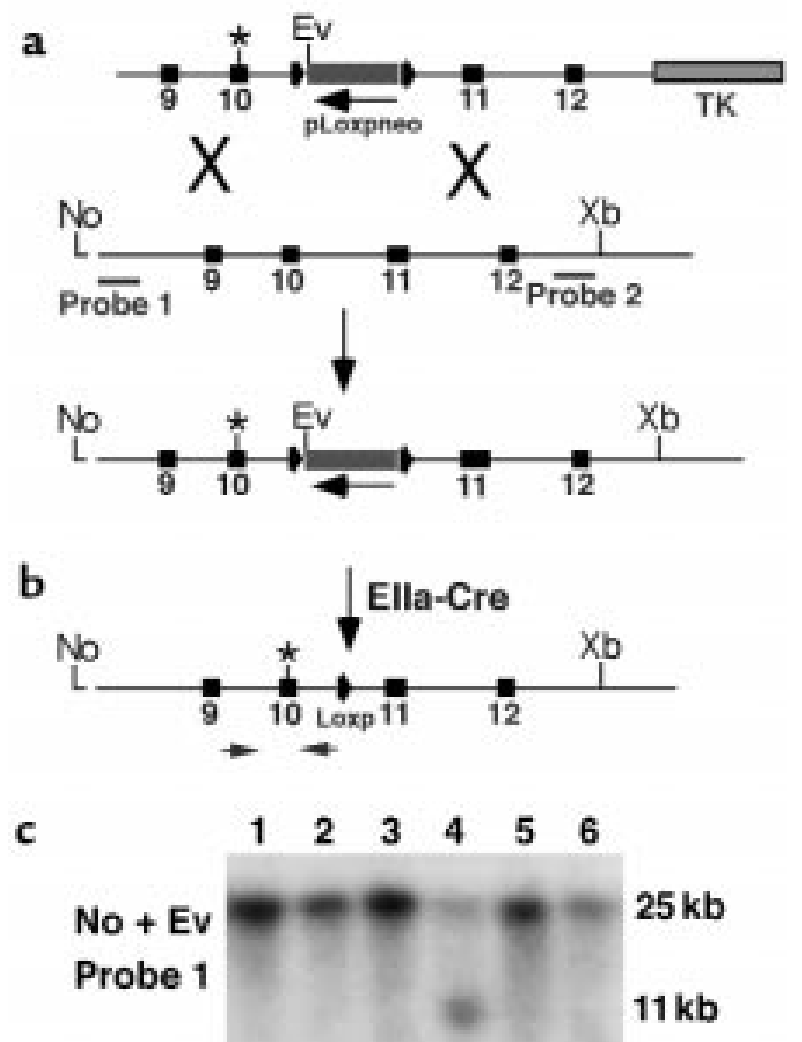

\section{Figure 2}

Ligand-independent dimerization and activation of G375C mutant FGFR3. (a) Wild type, G380R, S371C, G375C and K650E mutants of FGFR3 were transiently expressed in 293T cells in the absence or presence of $50 \mathrm{ng} / \mathrm{ml} \mathrm{aFGF}$, lysed and immunoprecipitated with anti FGFR3 antibody. Blots were developed using anti-FGFR3 antiserum (upper panel) or anti-phosphotyrosine antibody (lower panel). (b) Receptor phosphotyrosine activity normalized for expression levels ofFGFR3. In the absence of aFGF, FGFR3 mutants corresponding to the immunoblot above are phosphorylated. The K650E mutant has the highest basal activity, the G371C mutant is lower in basal activity but still higher than G375C and both are significantly higher than that of the wild type receptor. (c) Wild type and mutant FGFR3 transiently expressed in 293T cells were subjected to chemical cross-linking after being stimulated with $50 \mathrm{ng} / \mathrm{ml} \mathrm{aFGF}$. Ligand-independent receptor dimers were found for the S371C and G375C mutants, but not in the wild-type receptor.

ure $3, \mathrm{a}-\mathrm{c})$. F2 offspring were generated by intercrossing $\mathrm{F} 1$ heterozygous mice. At birth, both heterozygous $\left(\mathrm{Fgfr}^{369 /+}\right)$ and homozygous $\left(\mathrm{Fg} f \mathrm{r}^{369 / 369}\right)$ mutant mice were phenotypically normal (data not shown) but exhibited pronounced dwarfism during postnatal development. Tails of mutant mice grew slowly and stayed about $60-70 \%$ of the control at most time points measured (Figure 3c). The heterozygous mice exhibited intermediate lengths between the wild-type control and the homozygous ACH mice (Figure 3c). Dramatically reduced tail length suggests retarded growth of the long bones in mutant mice. A quantitative measurement of several major bones of the skeleton, prepared at multiple developmental stages, indicated that this indeed is the case. At postnatal day 15 (P15), the homozygous femurs, humeri, and vertebrae averaged 

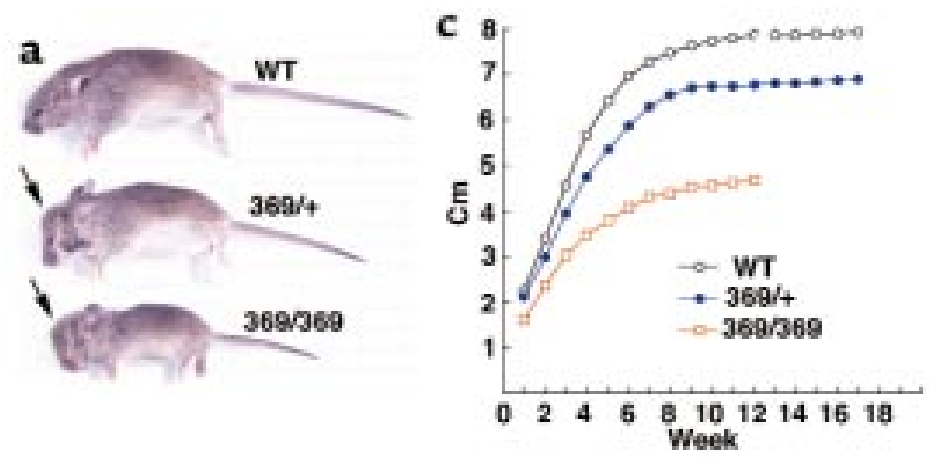

Figure 3

The Gly369Cys mutation in mouse FGFR3 results in dwarf mice. (a) Morphology and (b) skeleton of 21 day old wildtype (WT), Fgfr3 $3^{369 /+}(369 /+)$, and Fgfr3 $369 / 369(369 / 369)$ mice. Notice dome-shaped heads in 369/+ and 369/369 mice (arrows). (c) Quantitative measurements of tail length of mice with 3 genotypes. Each point represents data from an average of 5 mice. Standard deviation is less than $\pm 5 \%$. (d) X-ray images of $369 / 369$ and WT mice. Notice the reduced bone density in 369/369 mice: $F$, femur; $T$, tibia.


$60 \%, 56 \%$, and $85 \%$ of the lengths of wild-type littermates, respectively. Mutant mice also exhibited reduced bone density (Figure 3d). These observations indicated that the activation of FGFR3 by Gly369Cys mutation results in skeletal dysplasia in mice.

Retarded growth of cranial base in mutant mice results in macrocephaly. Patients with ACH exhibit macrocephaly (3). Similar to human patients, both Fgfr $3^{369 /+}$ and Fgfr $3^{369 / 369}$ mice had dome-shaped heads (Figure 3, a and $\mathrm{b}$ ). This phenotype became apparent at P7. At all developmental stages examined, skulls of Fgfr $3^{369 / 369}$ mice were more severely affected than those of heterozygous mice (Figure $4 \mathrm{a}$ ). The mutant skulls were markedly reduced in size along the anterior-posterior axis, whereas the left-right and dorsal-ventral axes were only slightly increased in mutant mice (Figure 4a, and unpublished observations). The bones of the skull are formed by both intramembranous and endochondral ossification. Macrocephaly seen in ACH is generally believed to be the consequence of an imbalance between these 2 modes of bone formation. To evaluate this in our mice, we examined 3 cranial base bones: occipital, basosphenoid, and presphenoid (Figure 4b), which are formed by endochondral ossification. Histological analysis of the P15-21 cranial base indicated that wild-type synchondroses were still in the cartilage state (Figure $4 d$ ), composed predominantly of proliferating and mature chondrocytes as revealed by Fgfr3 expression (Figure 4e) and hypertrophic chondrocytes as shown by ColX expression (Figure 4f). In contrast, the mutant synchondroses had prematurely fused and ossified (Figure 4, c and g), resulting in the much shorter cranial base in the mutant skulls (Figure 4c). Histological examination of synchondroses of P5 mice did not reveal any apparent difference between mutant and wild-type mice (data not shown). Beginning from P6, synchondroses of these bones in the mutant mice, on the other hand, initiated an accelerated process of closure and replacement by bone (Figure 4, h-j). We also found that the relative size of the mutant presphenoid was much smaller than the other 2 bones, indicating that it is most severely affected by the mutation (Figure $4 c)$. In contrast, the dimensions of the flat bones in the skull, which are formed by intramembranous ossification, were virtually unaffected in the mutant mice (data not shown). Thus, the dramatic shortening of cranial base, in concert with relatively normal flat bones, may serve as the major reason for the abnormal shape of the skull in the mutant mice.

FGFR3 Gly369Cys mutation activates Stats and cell-cycle inhibitors. Growth of long bones is primarily dependent on growth plate activity. We therefore compared growth plates from wild-type, Fgfr $3^{369 /+}$, and $F g f r 3^{369 / 369}$ mice. Growth-plate chondrocytes are typically divided into 4 types, i.e. resting, proliferating, maturation or prehypertrophic, and hypertrophic (Figure 5a). In Fgfr3 369/369 growth plates, the proliferation and maturation zones were disorganized and failed to form long chondrocyte columns (Figure 5c). Mutant growth plates also showed a dramatically expanded resting zone and significantly decreased maturation and hy-pertrophic zones compared with those of wild-type (Figure 5, a and c). Mutant mice also showed delayed formation of the secondary ossification center (Figure 5, a-c); this may partly account for the observed expansion of the resting zone. Heterozygous mice exhibited an intermediate phenotype between wild-type and Fgfr $3^{369 / 369}$ mice (Figure 5b).

Proliferation of chondrocytes in the growth plate was examined by $\left[{ }^{3} \mathrm{H}\right]$ thymidine incorporation. In wild-type mice, radiopositive signals were mainly localized within the proliferating zone and were much stronger than in mutant growth plates (Figure 5d). In Fgfr3369/369 growth plates, radiopositive cells were not only fewer in number but were also scattered throughout the growth 
plates (Figure 5f). At the age of about 6 weeks, there was virtually no detectable $\left[{ }^{3} \mathrm{H}\right]$ thymidine incorporation in Fgfr $3^{369 / 369}$ growth plate even after 4 hours of labeling, with heterozygous mice showing fewer radiopositive cells than wild-types (data not shown).

Activated FGF receptor signals cause activation of Stat proteins and cell-cycle inhibitors, both in vitro and in vivo (22-24). To determine whether the FGFR3 Gly369Cys mutation has a similar effect, we examined expression of these molecules in both mutant and normal growth plates using immunohistochemistry. We found that mutant growth plates exhibited increased intensities of FGFR3 (Figure 5, h and i), suggesting that the Gly369Cys FGFR3 may be more stable than wildtype protein. Notably, as the dosage of the FGFR3 mutation increased from heterozygous to homozygous, mutant growth plates showed increased levels of Stat1, Stat $5 \mathrm{a}$, and Stat $5 \mathrm{~b}$ proteins (Figure 5, $\mathrm{k}$ and $\mathrm{l}$; and unpublished observations). Nuclear translocation of Stat proteins is an accepted indicator of Stat activation; unphosphorylated Stat proteins are in the cytoplasm, and the activated forms are translocated into the nucleus $(22,23)$. Close examination revealed nuclear localization of Stat proteins in many mutant cells (Figure 5, $\mathrm{m}-\mathrm{O}$ ), suggesting that these proteins were activated.
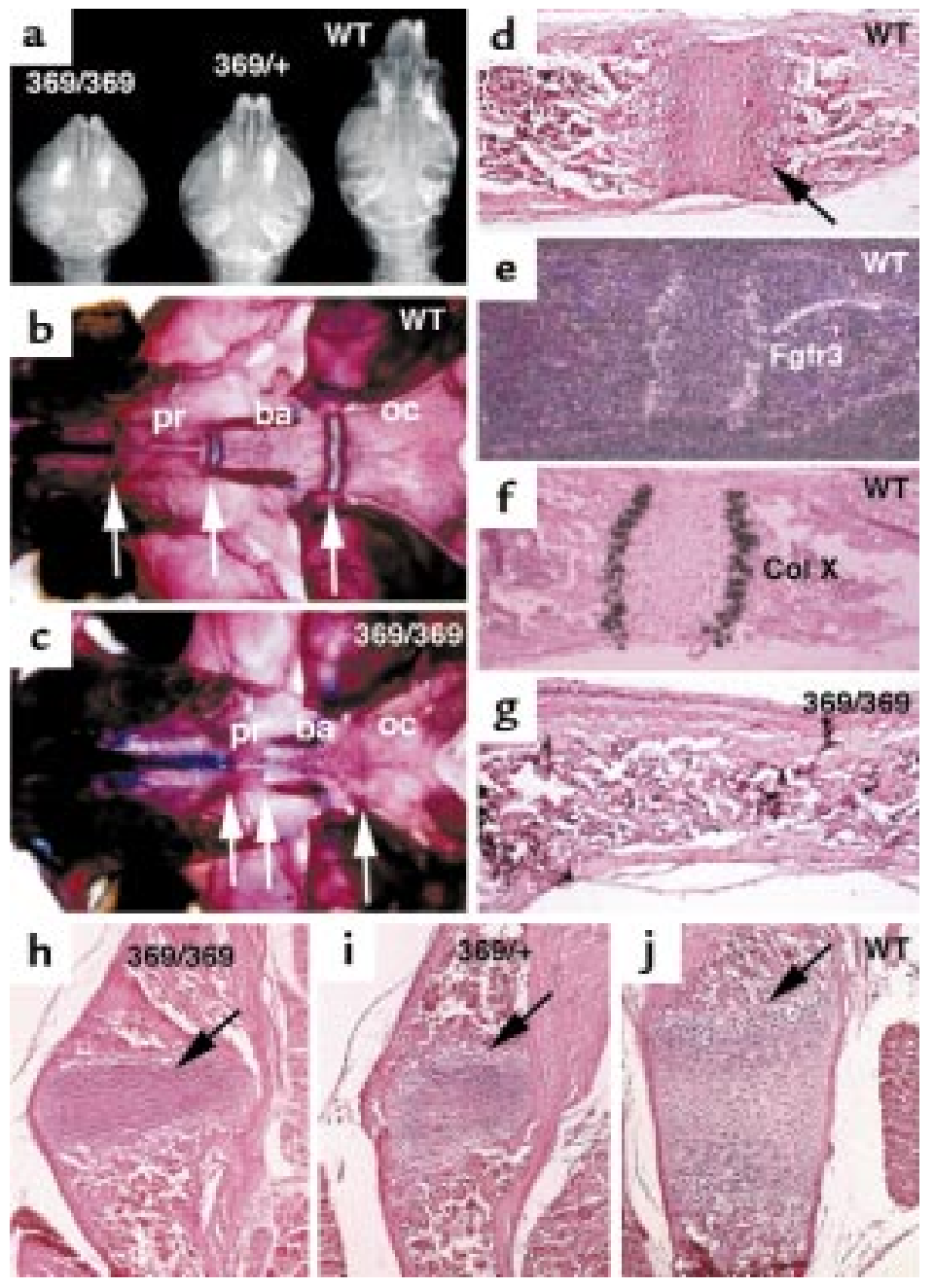

Mutant growth plates also exhibited increased staining for cell-cycle inhibitors including P16 and P19 (Figure 5 , $\mathrm{q}$ and $\mathrm{r}$; and unpublished observations). In wildtype mice, staining for these molecules is weak and mainly localized in hypertrophic and resting zones (Figure 5p). In mutant mice, however, the staining was significantly stronger and appeared more frequently in proliferating zones, especially in the flanking regions. In the central regions, the staining was relatively weak (Figure 5, $\mathrm{q}$ and $\mathrm{r}$ ).

The Gly369Cys mutation enhances osteoblast differentiation. Abnormal chondrogenesis may affect osteogenesis because long bone development is a coordinated process involving both chondrogenesis and osteogenesis. In the P15 mutant mice, the long bones had less primary spongiosa and less $\left[{ }^{3} \mathrm{H}\right]$ thymidine incorporation in the metaphyseal bone tissue than in wild-type littermates (Figure 5, d-f). Examination of mutant mice revealed an enhanced activity of osteoclasts, as demonstrated by increased TRAP staining at the interface between hypertrophic chondrocytes and trabecular bone (Figure 6, a-c). Staining undecalcified stage P1 knee joints with Alizarin Red S, a dye for mineralized tissues, revealed an advanced bone collar flanking the mutant, but not the wild-type growth plates (Figure 6, d-f). These observations, together with the premature ossification of synchondroses shown earlier (Figure 4), indicate that the Gly369Cys mutation results in advanced osteogenesis. To further our molecular understanding, we checked the expression of several genes that are intimately associated with osteoblast differentiation. We found increased expression levels of osteopontin (Figure 6, g-i), osteonectin (Figure 6, $\mathrm{j}-\mathrm{l}$ ), and osteocalcin (Figure $6, \mathrm{~m}-\mathrm{o}$ ) in the trabeculae of mutant long bones. Interestingly, osteocalcin not only is expressed at higher levels in the mutant mice, but is also ectopically expressed in the prehypertrophic region as revealed by in situ

\section{Figure 4}

Macrocephaly results from retarded growth of the cranial base. (a) Dorsal view of $x$-ray images of skulls of P21 day old $369 / 369,369 /+$, and WT mice. (b) Cranial base of WT, and (c) 369/369 mice. Arrows point to synchondroses: basooccipital (oc), basosphenoid (ba) and presphenoid (pr). Notice the absence of synchondroses in 369/369 mice (arrows in c). (d) H\&E image of a WT synchondrosis, which exhibits typical growth plate structures, including proliferating and maturing chondrocytes [positive for Fofr3 (e)], and hypertrophic chondrocytes [positive for collagen type X(f)]. (g) H\&E image of corresponding region of a 369/369 synchondrosis, which had completely turned into bone. (h-j) P6 synchondroses (arrows) of 369/369 (h), 369/+ (i) and WT (j) mice. The 369/369 synchondrosis was much thinner than the WT synchondrosis and was in a process of resolving, while the $369 /+$ synchondrosis (i) exhibited an intermediate phenotype between those of 369/369 and WT. 




hybridization (Figure 6, m-o), and immunohistochemical staining using an antibody to mouse osteocalcin (Figure 6, p-r).

\section{Discussion}

Numerous mutations in FGFR1-3 have been found to cause at least 9 human skeletal dysplasias (2-4). These mutations are thought to result in increased FGF/FGFR signals, as several studies showed ligandindependent activation of these mutant FGF receptors in vitro (25-29). Consistently, the overexpression of FGFs in mice has resulted in skeletal conditions that mimic human disease $(30,31)$. FGF receptors normally exist as inactive monomers and become activated upon ligand-triggered dimerization. Because many mutations

\section{Figure 5}

Histology and immunohistochemistry analyses of growth plates of P15 mice. Genotypes and markers were as indicated. (a) Chondrocytes in the WT growth plates are divided into 4 distinct zones, i.e. resting $(\mathrm{Rc})$, proliferating $(\mathrm{Pc})$, maturing $(\mathrm{Mc})$ and hypertrophic $(\mathrm{Hc})$ chondrocytes. (b and $\mathbf{c})$ In the mutant growth plates, the demarcation of each zone is not clear. Arrows point to the secondary ossification center. (d-f) $\left[{ }^{3} \mathrm{H}\right]$ Thymidine incorporation. The labeled cells in WT mark the Pc zone. In mutant growth plates, the labeled cells are fewer in number and scattered, suggesting that the majority of the cells are in a quiescent state. (g-o) Immunohistochemical analysis of growth plates, using antibodies to FGFR3 ( $\mathbf{g}-\mathbf{i})$, Stat $5 \mathrm{~b}$ (j-l, and $\mathbf{m}$-o for higher magnification), and p19 (p-r). In all cases, the staining was the weakest in WT, intermediate in $369 /+$, and strongest in 369/369 growth plates.

involved either gain or loss of a cysteine in the extracellular domain of FGF receptors, it has been postulated that the unpaired cysteine might promote intermolecular disulfide bond formation, leading to dimerization of the mutant receptors (32). Our observation that both Gly375Cys and Ser371Cys mutations caused ligand-independent dimerization directly supports this hypothesis.

The effects of activated FGFR3 signals on bone growth have been tested in vivo recently by using transgenic (12), and cDNA knock-in (23) models. The former used the type II collagen promoter and enhancer to control Fgfr3 Gly380Arg transgene expression, and the latter knocked a FGFR3 cDNA containing the Lys644Glu mutation, which results in TDII in human, into the endogenous Fgfr3 locus. Mice with retarded long bone growth were generated by both studies, although the mutant alleles of FGFR3 were not expressed faithfully. The ectopic expression of the Fgfr3 Gly380Arg transgene resulted in bone defects that were not observed in human achondroplasia (12), and the reduced expression of the Fgfr3 Lys644Glu allele did not cause the expected neonatal lethality seen in human patients with TD (23). Moreover, the existence of multiple splicing variants in the Fgfr3 locus (33) makes it impossible to mimic the endogenous situation, as only 1 isoform of FGFR3 was mutated by the approaches described here. To overcome these difficulties, we introduced a point mutation into the Fgfr 3 locus using a cotransfer approach (20) followed by the excision of the neo gene (Figure 1). The mutant allele was expressed faithfully after removal of the neo gene. A similar approach was used by a more recent study to introduce the Gly380Arg mutation into FGFR3 (11). Although this study showed that the Gly380Arg mutation results in dwarf mice, the molecular mechanism through which the mutation retards bone growth remains unclear. Of note, mutant mice displayed much more severe skeletal dysplasia than that exhibited by both Gly369Cys mice and human Gly380Arg patients. It is not known whether such a 
discrepancy between the Gly380Arg mice and Gly380Arg patients reflects an intrinsic difference between 2 species or is due to other unknown factors.

Our analysis of the mutant mice indicated that the Gly369Cys mutation affects chondrogenesis by restraining chondrocyte proliferation. It has been suggested that FGFR3 is a negative regulator of bone growth (15). This is primarily based on observations that loss of FGFR3 results in faster and prolonged bone growth $(15,21)$. One mechanism by which FGFR3 controls bone growth is by regulating signal transduction pathways through Stats, as demonstrated recently by the finding that FGFR3 carrying a TD II mutation caused activation of Stats and upregulation of cell-cycle inhibitors both in vitro and in vivo $(22,23)$. The observation that an achondroplasia mutation also causes activation of Stats and cell-cycle inhibitors suggests that the involvement of Stats and cell cycle proteins is a general phenomenon.

The markedly reduced sizes of mature and hypertrophic zones in mutant mice resemble phenotypes displayed by PTHrP or PTHrP-R knockout mice $(34,35)$. Ihb and PTHrP constitute a reciprocal regulation loop that controls chondrocyte proliferation and differentiation (36). We found that the expression domains and intensities of both PTHrP-R and Ibh were decreased in the Fofr $3^{369 / 369}$ growth plates (data not shown). This is consistent with recent studies showing that activation (23) or overexpression (12) of Fgfr 3 results in downregulation of $I h h$, supporting the notion that FGFR3 functions upstream of $I h b$ and negatively regulates its activity.

In our ACH mice, retarded bone growth is accompanied by advanced ossification as manifested by premature closure of cranial synchondroses and early onset of the bone collar flanking long bone growth plates. Because chondrogenesis and osteogenesis are coupled processes during endochondral bone formation, we have considered the possibility that the advanced ossification is secondary to the decreased chondrogenesis in mutant mice. However, this option is difficult to reconcile with some observations. For example, the advanced bone collar was observed in P1 (Figure 6, d-f) and older mutant mice, whereas the decreased proliferation of growth plate chondrocytes became obvious only after P7, arguing that the advanced bone collar is not merely a consequence of the decreased chondrogenesis. The advanced ossification can result in bone dysplasia because it does not allow sufficient time for chondrocytes and osteoblasts to proliferate. Our molecular analysis of mutant mice revealed increased expression of osteopontin, osteonectin, and osteocalcin, which are associated with the mature osteoblast differentiation stage (37-40), indicating that the Gly369Cys mutation enhances differentiation of osteoblasts. Enhanced differentiation is correlated with diminished osteoblast proliferation (41), which was observed in our mice. Osteopontin is likely to play a role in the early formative stages of osteogenesis. Phosphorylated osteopontin enhances attachment of osteoclasts to the bone matrix during bone resorption (42). Thus, the elevated expression of osteopontin may be 1 factor causing

\section{Figure 6}

The Gly369Cys mutation results in enhanced differentiation of osteoblast cells. Panel $\mathbf{a}-\mathbf{c}, \mathbf{g}-\mathbf{r}$ are prepared from P15 mice and panel $\mathbf{d}-\mathbf{f}$ from P1 mice. (a-c) Enhanced TRAP staining at the interface between mutant hypertrophic chondrocytes and primary spongiosa (arrows); the mutant also showed less primary spongiosa. (d-f) Alizarin Red S staining of undecalcified growth plates isolated from the knee joints of P1 mice. Arrows in (e) and (f) point to the advanced bone collars. (g-o) In situ hybridization using probes for osteopontin (Op, g-i), osteonectin (On, $\mathbf{j}-\mathbf{I}$ ) and osteocalcin (Oc, $\mathbf{m}-\mathbf{o})$. (p-r) Immunohistochemical staining of osteocalcin. Arrows in $\mathbf{n}$ and $\mathbf{o}$ point to ectopic expression of $\mathrm{Oc}$ in the maturing zone of chondrocytes. Both Op and On are also stronger in the $369 /+$ and 369/369 trabecular bones. 
increased activity of osteoclasts in mutant growth plates. Osteocalcin is an osteoblast-specific protein expressed mainly in cortical and trabecular bone (43). In mutant mice, however, osteocalcin was abnormally expressed in all prehypertrophic chondrocytes.

The consequence of the ectopic expression of osteocalcin in the prehypertrophic zone of chondrocytes is not clear. Expression of osteocalcin is associated with osteoblast differentiation (41); thus, the ectopic expression of osteocalcin may presage abnormal differentiation of prehypertrophic chondrocytes into osteoblastlike cells, a process termed transdifferentiation that is sometimes observed in chicken (44). Osteocalcin is inferred to be a negative regulator of bone formation, based on the increased bone mass and accelerated bone growth caused by the null mutation (45). Because the $\mathrm{ACH}$ mice exhibited retarded bone growth and reduced bone density, we believe that increased and ectopic expression of osteocalcin by the activated FGFR3 signals is partially responsible for this phenotype. The reduced bone density, which is reflected by the reduced number and size of trabeculae, could also be caused by increased osteoclast activity (TRAP, Figure 6a) and/or decreased chondrogenesis. The mutant growth plate contains fewer clones of hypertrophic cells and fewer longitudinal septa between these clones. As the ossification front advances, fewer subchondral trabeculae will be left behind.

In summary, we have shown that the Gly375Cys mutation activates FGFR3 by inducing ligand-independent dimerization of the receptor. When this mutation is introduced into mouse, it results in dwarfism sharing similar phenotypes with human achondroplasia. Molecular analysis reveals activation of Stat proteins and cell-cycle inhibitors, which may be responsible for the decreased proliferation of mutant chondrocytes. The activation of FGFR3 also results in downregulation of $I h b$ and $P T H r P-R$ and upregulation of osteoblast differentiation markers, as evidence for both abnormal chondrogenesis and osteogenesis. These data reveal an essential role of FGF/FGFR3 signals in both chondrogenesis and osteogenesis during endochondral ossification. The dwarf mouse model offers a valuable tool for further exploration of the signaling pathways that regulate normal bone growth.

\section{Acknowledgments}

We thank the Press Family Foundation for their generous and continuous support to this project. We thank J. Gotay, J. Xie, and X. Xu for technical support, and M. Weinstein for critical reading of the manuscript. This work was supported in part by the Israel Academy of Sciences and by ProChon Biotech Ltd. A. Yayon is incumbent of the Alvin and Gertrude Levine Career Development Chair of Cancer Research.

\footnotetext{
1. Gilbert, S.F. 1994. The development of bones. In Developmental biology. 4th edition. S.F. Gilbert, editor. Sinauer Associates. Sunderland, MA. 333-338.

2. Horton, W.A. 1997. Fibroblast growth factor receptor 3 and the human chondrodysplasias. Curr. Opin. Pediatr. 9:437-442.
}

3. Muenke, M., and Schell, U. 1995. Fibroblast-growth-factor receptor mutations in human skeletal disorders. Trends Genet. 11:308-313.

4. Mulvihill, J.J. 1995. Craniofacial syndromes: no such thing as a single gene disease. Nat. Genet. 9:101-103.

5. Rousseau, F., et al. 1994. Mutations in the gene encoding fibroblast growth factor receptor-3 in achondroplasia. Nature. 371:252-254.

6. Shiang, R., et al. 1994. Mutations in the transmembrane domain of FGFR3 cause the most common genetic form of dwarfism, achondroplasia. Cell. 78:335-342.

7. Tavormina, P.L., et al. 1997. Thanatophoric dysplasia (types I and II) caused by distinct mutations in fibroblast growth factor receptor 3. Nat. Genet. 9:321-328.

8. Bellus, G.A., et al. 1995. A recurrent mutation in the tyrosine kinase domain of fibroblast growth factor receptor 3 causes hypochondroplasia. Nat. Genet. 10:357-359.

9. Oberklaid, F., Danks, D.M., Jensen, F., Stace, L., and Rosshandler, S. 1979. Achondroplasia and hypochondroplasia. Comments on frequency, mutation rate, and radiological features in skull and spine. J. Med. Genet. 16:140-146.

10. Superti-Furga, A., et al. 1995. A glycine 375-to-cysteine substitution in the transmembrane domain of the fibroblast growth factor receptor-3 in a newborn with achondroplasia. Eur. J. Pediatr. 154:215-219.

11. Wang, Y., et al. 1999. A mouse model for achondroplasia produced by targeting fibroblast growth factor receptor. Proc. Natl. Acad. Sci. USA. 96: $4455-4460$

12. Naski, M.C., Colvin, J.S., Coffin, J.D., and Ornitz, D.M. 1998. Repression of hedgehog signaling and BMP4 expression in growth plate cartilage by fibroblast growth factor receptor 3. Development. 125:4977-4988.

13. Webster, M.K., and Donoghue, D.J. 1996. Constitutive activation of fibroblast growth factor receptor 3 by the transmembrane domain point mutation found in achondroplasia. EMBOJ. 15:520-527.

14. Li, Y., Mangasarian, K., Mansukhani, A., and Basilico, C. 1997. Activation of FGF receptors by mutations in the transmembrane domain. Oncogene. 27:1397-1406.

15. Deng, C., Wynshaw-Boris, A., Zhou, F., Kuo, A., and Leder, P. 1996. Fibroblast growth factor receptor 3 is a negative regulator of bone growth. Cell. 84:911-921.

16. Yang, X., Li, C., Xu, X., and Deng, C. 1998. The tumor suppressor SMAD4/DPC4 is essential for epiblast proliferation and mesoderm induction in mice. Proc. Natl. Acad. Sci. USA. 95:3667-3672.

17. Lakso, M., et al. 1996. Efficient in vivo manipulation of mouse genomic sequences at the zygote stage. Proc. Natl. Acad. Sci. USA. 93: 5860-5865.

18. Albrecht, U., Eichele, G., Helms, J.A., and Lu, H-C. 1997. Visualization of gene expression patterns by In Situ hybridization. In Molecular and cellular methods in developmental toxicology. George P. Daston, editor. CRC Press. Boca Raton, FL. 49-69.

19. Ornitz, D.M., and Leder, P. 1992. Ligand specificity and dependence of fibroblast growth factor receptors 1 and 3. J. Biol. Chem. 267:16305-16311.

20. Deng, C., Thomas, K.R., and Capecchi, M.R. 1993. Location of crossovers during gene targeting with insertion and replacement vectors. Mol. Cell. Biol. 13:2134-2140.

21. Colvin, J.S., Bohne, B.A., Harding, G.W., McEwen, D.G., and Ornitz, D.M. 1996. Skeletal overgrowth and deafness in mice lacking fibroblast growth factor receptor 3. Nat. Genet. 12:390-397.

22. Su, W.C., et al. 1997. Activation of Stat 1 by mutant fibroblast growthfactor receptor in thanatophoric dysplasia type II dwarfism. Nature. 386:288-292.

23. Li, C., et al. 1999. A Lys644Glu substitution in fibroblast growth factor receptor 3 (FGFR3) causes dwarfism in mice by activation of STATs and ink 4 cell cycle inhibitors. Hum. Mol. Genet. 8:35-44.

24. Sahni, M., et al. 1999. FGF signaling inhibits chondrocyte proliferation and regulates bone development through the STAT-1 pathway. Genes Dev. 13:1361-1366.

25. Neilson, K.M., and Friesel, R.E. 1995. Constitutive activation of fibroblast growth factor receptor- 2 by a point mutation associated with Crouzon syndrome. J. Biol. Chem. 270:26037-26040.

26. Neilson, K.M., and Friesel, R. 1996. Ligand-independent activation of fibroblast growth factor receptors by point mutations in the extracellular, transmembrane, and kinase domains. J. Biol. Chem. 271:25049-25057.

27. Robertson, S.C., et al. 1998. Activating mutations in the extracellular domain of the fibroblast growth factor receptor 2 function by disruption of the disulfide bond in the third immunoglobulin-like domain. Proc. Natl. Acad. Sci. USA. 95:4567-4572.

28. Galvin, B.D., Hart, K.C., Meyer, A.N., and Webster, M.K. 1996. Constitutive receptor activation by Crouzon syndrome mutations in fibroblast growth factor receptor (FGFR)2 and FGFR2/Neu chimeras. Proc. Natl. Acad. Sci. USA 93:7894-7899.

29. Park, W.J., et al. 1995. Analysis of phenotypic features and FGFR2 mutations in Apert syndrome. Am. J. Hum. Genet. 57:321-328.

30. Coffin, J.D., et al. 1995. Abnormal bone growth and selective translational regulation in basic fibroblast growth factor (FGF-2) transgenic mice. Mol. Biol. Cell. 6:1861-1873.

31. Carlton, M.B., Colledge, W.H., and Evans, M.J. 1998. Crouzon-like cran- 
iofacial dysmorphology in the mouse is caused by an insertional mutation at the Fgf3/Fgf4 locus. Dev. Dyn. 212:242-249.

32. Wilkie, A.O., Morriss-Kay, G.M., Jones, E.Y., and Heath, J.K. 1995. Functions of fibroblast growth factors and their receptors. Curr. Biol. 5:500-507.

33. Givol, D., and Yayon, A. 1992. Complexity of FGF receptors: genetic basis for structural diversity and functional specificity. FASEB J. 6:3362-3369.

34. Karaplis, A.C., et al. 1994. Lethal skeletal dysplasia from targeted disruption of the parathyroid hormone-related peptide gene. Genes Dev. 8:277-289.

35. Lanske, B., et al. 1996. PTH/PTHrP receptor in early develop ment and Indian hedgehog-regulated bone growth. Science. 273:663-666

36. Vortkamp, A., et al. 1996. Regulation of rate of cartilage differentiation by Indian hedgehog and PTH-related protein. Science. 273:613-622.

37. Erlebacher, A., Filvaroff, E.H., Gitelman, S.E., and Derynck, R. 1995. Toward a molecular understanding of skeletal development. Cell. 80:371-378.

38. Komori, T., and Kishimoto, T. 1998. Cbfa1 in bone development. Curr. Opin. Genet. Dev. 8:494-499.

39. Komori, T., et al. 1997. Targeted disruption of Cbfa1 results in a com- plete lack of bone formation owing to maturational arrest of osteoblasts. Cell. 89:755-764.

40. Ducy, P., Zhang, R., Geoffroy, V., Ridall, A.L., and Karsenty, G. 1997. Osf2/Cbfa1: a transcriptional activator of osteoblast differentiation. Cell. 89:747-754

41. Lian, J.B., Stein, G.S., Stein, J.L., and van Wijnen, A.J. 1998. Osteocalcin gene promoter: unlocking the secrets for regulation of osteoblast growth and differentiation. J. Cell. Biochem. Suppl. 30-31:62-72.

42. Denhardt, D.T., and Noda, M. 1998. Osteopontin expression and function: role in bone remodeling. J. Cell. Biochem. Suppl. 30-31:92-102.

43. Nakase, T., et al. 1994. Alterations in the expression of osteonectin, osteopontin and osteocalcin mRNAs during the development of skeletal tissues in vivo. Bone Miner. 26:109-122.

44. Cancedda, R., Descalzi Cancedda, F., and Castagnola, P. 1995. Chondrocyte differentiation. Int. Rev. Cytol. 159:265-358.

45. Ducy, P., et al. 1996. Increased bone formation in osteocalcin-deficient mice. Nature. 382:448-452. 